\title{
Analyse phytochimique et évaluation de la toxicité aiguë par voie orale chez des rats de l'extrait total aqueux des feuilles de Abrus precatorius Linn (Fabaceae)
}

\author{
Marius LEBRI ${ }^{1,5}$ *, Calixte BAHI ${ }^{1}$, N'guéssan Bra Yvette FOFIE $^{2}$, Goueh GNAHOUE ${ }^{3}$, \\ Stéphanie Marianne LAGOU ${ }^{4}$, Hanane ACHIBAT ${ }^{5}$, Ahoua YAPI $^{6}$, Guédé Noel ZIRIHI ${ }^{7}$, \\ Adama COULIBALY ${ }^{1}$, Abderrafia HAFID ${ }^{5}$ et Mostafa KHOUILI ${ }^{5}$ \\ ${ }^{1}$ Laboratoire de Pharmacodynamie Biochimique, UFR Biosciences, \\ Université Félix Houphouet Boigny, 22 BP 582 Abidjan 22, Côte d'Ivoire. \\ ${ }^{2}$ Laboratoire de Pharmacognosie, Botanique et Cryptogamie, UFR Sciences Pharmaceutique et Biologiques, \\ Université Félix Houphouët Boigny, 22 B.P.747 Abidjan 22, Côte d'Ivoire. \\ ${ }^{3}$ Laboratoire de Biochimie (Sciences de la Vie et de la Terre), \\ Ecole Normale 02 BP 801 Abidjan 02, Côte d'Ivoire. \\ ${ }^{4}$ Laboratoire d'Ethnopharmacologie et de Plantes Médicinales, \\ Pôle de Recherche en Pharmacopée Africaine et Substance Naturelle UFR Science de la \\ Nature Biosciences, Université Nangui Abrogoua, 02 BP 801 Abidjan 02, Côte d'Ivoire. \\ ${ }^{5}$ Laboratoire de Chimie Organique et Analytique, Equipe de Chimie Organique et \\ Organométallique Appliquées, Université Sultan Moulay Slimane, \\ Faculté des Sciences et Techniques, BP 523, 23000 Béni-Mellal, Maroc. \\ ${ }^{6}$ Laboratoire de Zoologie et Biologie Animale, UFR Biosciences, \\ Université Felix Houphouet Boigny, 22 BP 582 Abidjan 22, Côte d'Ivoire. \\ ${ }^{7}$ Laboratoire de Botanique, UFR Biosciences, Université Félix Houphouët Boigny, \\ Cote d'Ivoire22 BP 582 Abidjan 22, Côte d'Ivoire. \\ *Auteur correspondant, E-mail : lebrimarius7@ gmail.com; \\ Tel : (212) 5234851 12/22/82, Fax: (212) 523485201
}

\section{RÉSUMÉ}

Abrus precatorius est reconnue en médecine traditionnelle pour ses nombreuses vertus thérapeutiques. En Côte d'Ivoire, les feuilles sont utilisées dans une boisson pour le traitement des troubles gynécoobstétriques. Au Nigeria, le décocté des feuilles est utilisé dans le traitement du diabète. Des travaux antérieurs sur la plante ont mis en évidence la présence de l'abrine qui est un composé chimique toxique contenu dans les feuilles et dans les graines. L'intérêt de l'utilisation des feuilles de Abrus precatorius exige qu'une approche de sa toxicité puisse être entreprise en vue d'évaluer son innocuité chez l'homme. La présente étude a consisté évaluer la toxicité aiguë par voie orale de l'extrait total aqueux des feuilles de Abrus precatorius (ETAAP) chez des rats. Une analyse phytochimique basée sur des réactions de coloration et/ou de précipitation a été effectué afin d'identifier les composés chimiques présent dans l'extrait. La Dose Létale $50\left(\mathrm{DL}_{50}\right)$ a été déterminée et les animaux ont été observés durant 14 jours après un gavage unique à des doses de $5 \mathrm{mg} / \mathrm{kg}, 50$ $\mathrm{mg} / \mathrm{kg}, 300 \mathrm{mg} / \mathrm{kg}, 2000 \mathrm{mg} / \mathrm{kg}$ et $5000 \mathrm{mg} / \mathrm{kg}$ de poids corporels (pc) contre un témoin. La DL $\mathrm{L}_{50}$ obtenue a été supérieure à $5000 \mathrm{mg} / \mathrm{kg}$ de pc, aucune mortalité et aucun signe clinique de toxicité n'a été observé chez tous les animaux. Cette étude révèle que l'ETAAP est non toxique par voie orale chez des rats. L'analyse phytochimique a montré une hétérogénéité de groupes chimiques (alcaloïdes, tanins, flavones, coumarines, saponines, stérols, triterpènes et des composés réducteurs) dont la présence dans l'extrait pourrait être responsable des propriétés thérapeutiques attribuées à Abrus precatorius.

(C) 2015 International Formulae Group. All rights reserved.

Mot clés : Abrus precatorius $\mathrm{L}$, médecine traditionnelle, innocuité, $\mathrm{DL}_{50}$. 


\section{INTRODUCTION}

Le genre Abrus Adans contient à peu près 17 espèces trouvées en Afrique, en Madagascar, en Inde et Indochine. Parmi ces espèces, Abrus precatorius Linn est probablement la plus connue, elle est retrouvée partout dans les tropiques et les géotropiques (Schrire, 2005; Prenner, 2013). Les feuilles de cette plante grimpante de la famille des Fabaceae, sont reconnues dans divers pays pour ses nombreuses vertus thérapeutiques en médecine traditionnelle. En Inde, ces feuilles sont utilisées pour guérir la fièvre, les troubles d'estomac, l'asthme et la bronchite (Kirtikar et Basu, 1987; Suralkar et Kasture, 2013). Les feuilles à goût sucré sont très prisées en Madagascar et au Sénégal dans le traitement de la toux, surtout la toux infantile. Ainsi, Abrus precatorius est classée parmi les plantes antitussives (Gairola et al., 2010). Au Nigeria, le décocté des feuilles est utilisé dans le traitement du diabète (Ezuruike et Prieto, 2014). En Côte d'Ivoire, une enquête ethnobotanique a montré que les feuilles de Abrus precatorius sont utilisées au Sud du pays, chez les peuples Abbey et Krobou d'Agboville, dans une boisson pour faciliter l'accouchement des femmes (N'guéssan et al., 2010).

Par ailleurs, des travaux ont révélé que les feuilles et les graines contiennent de l'abrine (Garanie, 2010) qui est un composé très toxique (Okoko et Yama, 2011). Une graine bien mastiquée peut causer l'empoisonnement total des adultes et des enfants (Okoko et Yama, 2011). Ces informations sur les manifestations cliniques multiples liées à l'exposition de l'homme à la plante, sont à l'origine de la présente étude.

Elle visait à évaluer la toxicité aiguë par voie orale de l'extrait total aqueux de feuilles de Abrus precatorius obtenue par la méthode traditionnelle (décoction) en déterminant la Dose Létale $50\left(\mathrm{DL}_{50}\right)$, tout en mettant un accent sur les différents signes cliniques de toxicité et des morts éventuelles. L'analyse phytochimique qualitative a permis de mettre en évidence les groupes chimiques qui seraient responsable de nombreuse propriétés thérapeutiques attribuées à cette plante.

\section{MATERIEL ET METHODES \\ Matériel végétal}

Les feuilles de Abrus precatorius ont été collectées dans les environs de la ville d'Abidjan en Côte d'Ivoire. La plante avait été déjà identifiée au Centre National d'Abidjan sur le numéro: Abrus precatorius (Fabaceae) : Aboudé-Mandéké (Côte d'Ivoire), 23 mai 1990, N'guessan Koffi 165 (N'guéssan et al., 2010). Les feuilles séchées à l'abri du soleil ont été broyées et la poudre obtenue a été utilisé pour la préparation de l'extrait.

\section{Matériel animal}

Les animaux expérimentaux sont des rats blancs mâles de souche Wistar âgés de 8 à 9 semaines dont le poids est compris entre 161-336 g. Les rats ont été placés dans des cages plastiques contenant des copeaux de bois renouvelés tous les 3 jours. Les 18 rats mâles ont été acclimatés aux conditions de laboratoire (une température de 20 à $22^{\circ} \mathrm{C}, 12$ heures de lumière et 12 heures d'obscurité) pendant 7 jours puis repartis en 6 lots homogènes de 3 . Ils ont été mis à jeun pendant $18 \mathrm{~h}$ avant l'administration de l'extrait par gavage. Les animaux ont été privés de nourriture mais pas d'eau. Ces animaux proviennent d'une ferme privée de la ville d'Abidjan (Côte d'Ivoire).

\section{Préparation de l'extrait totale aqueux des feuilles de Abrus precatorius}

L'extrait total aqueux des feuilles d'Abrus precatorius a été préparé en suivant la méthode préconisée en médecine traditionnelle (décoction) (Konkon et al., 2006). Pour cette étude expérimentale, les méthodes ont été adaptéee à la réalité du laboratoire. Pour cela, $100 \mathrm{~g}$ de poudre de feuilles ont été ajoutés dans 2 litres d'eau distillée dans un récipient adapté (marmite métallique). Le tout a été porté à ébullition 
pendant 15 minutes. La solution obtenue a été filtrée et le filtrat a été évaporé à l'étuve à la température de $50{ }^{\circ} \mathrm{C}$. L'extrait sec obtenu de couleur marron constitue l'extrait total aqueux des feuilles d'Abrus precatorius. L'opération a été répétée plusieurs fois afin d'obtenir une quantité d'extrait suffisante pour les expériences.

\section{Analyse phytochimique}

Les différentes analyses chimiques ont été effectuées, par criblage phyto-chimique, au sein du Laboratoire de Chimie Organique et Analytique (Équipe de Chimie Organique et Organométallique Appliquées), à la Faculté des Sciences et Techniques de l'Université Sultan Moulay Slimane de Béni-Mellal, Maroc.

Il s'agit d'une analyse qualitative basée sur des réactions de coloration et/ou de précipitation. (Houghton et Raman, 1998). $2 \mathrm{~g}$ de l'extrait ont été ajoutés à $50 \mathrm{ml}$ d'eau distillée pour reconstituer l'extrait total aqueux des feuilles d'Abrus precatorius.

Pour une analyse comparative, différents extraits de la feuille ont été préparé. $25 \mathrm{~g}$ de poudre de feuille ont été extrait successivement par $125 \mathrm{ml}$ d'éthanol, par 125 ml d'acétate d'éthyle et par $125 \mathrm{ml}$ d'hexane. Le Tableau 1 indique les différents groupes chimiques recherchés et les réactifs spécifiques utilisés.

\section{Toxicité aiguë par voie orale}

Cette étude expérimentale a été adaptée à celle décrite par la ligne directrice 423 (OCDE, 2001). Les concentrations de l'extrait total aqueux de Abrus precatorius ont été préparées sur la base du principe selon lequel les concentrations à administrer doivent être ramenées au poids corporel des rats, ainsi les doses ont été exprimées en $\mathrm{mg} / \mathrm{kg}$ de poids corporel. Une solution mère de l'extrait total aqueux d'Abrus precatorius a été obtenue à partir de la dissolution de $25 \mathrm{~g}$ de produits dans $100 \mathrm{ml} \mathrm{d}$ 'eau distillé soit $250 \mathrm{mg} / \mathrm{ml}$ ou une concentration de $25 \%$. A partir de cette solution mère, différentes dilutions ont été effectuées pour obtenir des concentrations de : 0,$25 ; 2,5 ; 15 ; 100 ; 250 \mathrm{mg} / \mathrm{ml}$, correspondants respectivement aux doses de : $5 \mathrm{mg} / \mathrm{kg}, 50$ $\mathrm{mg} / \mathrm{kg}, \quad 300 \mathrm{mg} / \mathrm{kg}, 2000 \mathrm{mg} / \mathrm{kg}$ et 5000 $\mathrm{mg} / \mathrm{kg}$. Les différents lots d'animaux ont été traités à différentes doses de l'ETAAP contre le témoin recevant de l'eau distillée.

Tableau 1: Réactifs spécifiques et réactions du criblage phytochimique.

\begin{tabular}{llll}
\hline Groupes chimiques & Réactifs spécifiques & Réactions caractéristiques \\
\hline Alcalö̈des & Tanins & $\begin{array}{l}\text { Dragendorff. (tétraiodo- } \\
\text { bismuthale de potassium) }\end{array}$ & $\begin{array}{l}\text { Coloration orangée avec } \\
\text { apparition de précipité }\end{array}$ \\
\hline \multirow{2}{*}{$\begin{array}{l}\text { Composés } \\
\text { polyphénoliques }\end{array}$} & Flavonoïdes & réaction à la cyanidine & $\begin{array}{l}\text { Une coloration verdâtre ou bleu- } \\
\text { noirâtre. }\end{array}$ \\
\cline { 2 - 4 } & Coumarines & $\begin{array}{l}\text { Coloration rose orangé; rose } \\
\text { violacé ou rouge }\end{array}$ \\
\hline composés quinoniques de Bornträger- UV & Inflorescence intense \\
\hline Saponines & & $\begin{array}{l}\text { Détermination de l'Indice } \\
\text { Mousse }\left(\mathrm{IM}{ }^{*}\right)\end{array}$ & $\begin{array}{l}\text { Test positif si IM>100 mousse } \\
\text { intense }\end{array}$ \\
\hline Stérols et triterpènes & & $\begin{array}{l}\text { Libermann-Burchard } \\
\left.\text { (Anhydride Acétique- } \mathrm{H}_{2} \mathrm{SO}_{4}\right)\end{array}$ & $\begin{array}{l}\text { L'apparition à l'interphase d'un } \\
\text { anneau pourpre ou violet, virant } \\
\text { au bleu puis au vert }\end{array}$ \\
\hline Composés réducteurs & & Test à la de liqueur de Fehling & Précipité rouge brique \\
\hline
\end{tabular}


Les animaux de chaque lot d'essai ont été gavés 1 à 1 avec un temps d'observation. Lorsque le lot traité ne présentait aucun mort et aucun signe de toxicité, le lot suivant était ensuite traité. Au terme de l'expérience, tous les lots ont été traités et les animaux ont été soumis à observation les premières $24 \mathrm{~h}$ et régulièrement pendant 14 jours.

\section{RESULTATS}

Les résultats de l'analyse phytochimique ont révélé que l'extrait aqueux, utilisé pour le test de toxicité, contenait 9 groupes de composés chimiques : les alcaloïdes, les tanins, les flavonoïdes (flavones), les saponines, les composés quinoniques (coumarines), les stérols et les triterpènes et les composés réducteurs (Tableau 2). La totalité des groupes chimiques ont été fortement caractérisés dans l'ETAAP comparativement aux autres extraits des feuilles obtenues à partir des solvants organiques. Les résultats montrent une forte présence des alcaloïdes l'extrait éthanolique comparativement aux stérols et triterpènes en faible présence. L'extrait acétatique montre une forte présence de tanins et de flavones comparativement aux stérols et triterpènes faiblement caractérisés. Les stérols et triterpènes sont fortement caractérisés dans l'extrait hexanique. Tous les autres groupes chimiques non mentionnés pour un extrait donné, n'ont pas été caractérisés lors des tests phytochimiques.

Les résultats de l'étude sur la toxicité aiguë par voie orale, ne montre aucun mort et aucun signe clinique de toxicité (Tableau 3) après l'administration des doses de 5, 50, 300, 2000 et $5000 \mathrm{mg} / \mathrm{kg}$ de poids corporel (pc) de l'extrait. Tous les animaux ont survécu à l'issue des 14 jours d'observation, ce qui implique que la $\mathrm{DL}_{50}$ est supérieure à 5000 $\mathrm{mg} / \mathrm{kg}$ de pc. Selon le Système Général Harmonisé de classification et d'étiquetage des produits chimiques (SGH, 2003), l'extrait total aqueux des feuilles d'Abrus precatorius est non toxique par voie orale chez des rats Wistar.

Tableau 2: Groupes chimiques présents dans les différents extraits des feuilles d'Abrus precatorius.

\begin{tabular}{llllll}
\hline \multirow{2}{*}{ Groupes chimiques } & \multicolumn{2}{l}{ Extraits } & & \\
\cline { 2 - 6 } & $\begin{array}{l}\text { Extrait total } \\
\text { aqueux }\end{array}$ & Ethanolique & Acétatique & Hexanique \\
\hline Alcaloïdes & +++ & +++ & - & - \\
\hline $\begin{array}{l}\text { Substances } \\
\text { phénoliques }\end{array}$ & Tanins & +++ & - & +++ & - \\
\cline { 2 - 6 } & $\begin{array}{l}\text { Flavonoïdes } \\
\text { (flavones) }\end{array}$ & +++ & - & +++ & - \\
\hline $\begin{array}{l}\text { composés } \\
\text { quinoniques }\end{array}$ & Coumarines & +++ & - & - & - \\
\hline Saponines & & +++ & - & - & - \\
\hline Stérols et triterpènes & +++ & + & + & +++ \\
\hline Composés réducteurs & +++ & - & & - \\
\hline \multicolumn{1}{c}{$+:$ présence. ; +++ : Intense présence $;-:$ absence } & & & \\
\hline
\end{tabular}


Tableau 3 : Résultats de l'observation des animaux durant les premières $24 \mathrm{~h}$ et tous les jours pendant 14 jours après administration orale de l'extrait total aqueux d'Abrus precatorius

\begin{tabular}{|c|c|c|c|c|c|c|}
\hline $\begin{array}{l}\text { Signes } \\
\text { cliniques de toxicité par lot } \\
\text { d'animaux }\end{array}$ & $\begin{array}{c}\text { Lot1 } \\
\text { (témoin) }\end{array}$ & $\begin{array}{c}\text { Lot2 } \\
5 \mathrm{~m} / \mathrm{kg} \\
\mathrm{pc}\end{array}$ & $\begin{array}{l}\text { Lot3 } \\
50 \mathrm{mg} / \\
\mathrm{kg} \mathrm{pc}\end{array}$ & $\begin{array}{l}\text { Lot4 } \\
300 \mathrm{mg} / \\
\text { kg pc }\end{array}$ & $\begin{array}{c}\text { Lot5 } \\
2000 \mathrm{mg} / \\
\mathrm{kg} \mathrm{pc}\end{array}$ & $\begin{array}{c}\text { Lot6 } \\
5000 \mathrm{mg} / \\
\mathrm{kg} \mathrm{pc}\end{array}$ \\
\hline Apathie & - & - & - & - & - & - \\
\hline Excitation & - & - & - & - & - & - \\
\hline Troubles de la respiration & - & - & - & - & - & - \\
\hline Refus de nourriture & - & - & - & - & - & - \\
\hline Saignement buccal & - & - & - & - & - & - \\
\hline Saignement nasal & - & - & - & - & - & - \\
\hline Douleur abdominale (contorsion) & - & - & - & - & - & - \\
\hline Coma & - & - & - & - & - & - \\
\hline Diarrhée & - & - & - & - & - & - \\
\hline Tremblement & - & - & - & - & - & - \\
\hline Convulsion & - & - & - & - & - & - \\
\hline Mortalité & - & - & - & - & - & - \\
\hline
\end{tabular}

+ : présence ; - : absence

\section{DISCUSSION}

L'étude phytochimique qualitative de l'extrait total aqueux des feuilles d'Abrus precatorius a montré que la plante contient des alcaloïdes, des tanins, des flavonoïdes (flavones), des saponines, des composés quinoniques (coumarines), des stérols, des triterpènes et des composés réducteurs. La totalité des groupes chimiques de la plante a été caractérisée dans l'ETAAP. La méthode d'extraction utilisée en médecine traditionnelle (décoction) est donc du point de vue qualitatif aussi efficace que les autres méthodes d'extraction étudiées (éthanolique, acétatique et hexanique). Des travaux effectués sur la plante ont permis d'isoler plusieurs groupes de composés secondaires dont les alcaloïdes, les stéroïdes et triterpènoïdes, les isoflavanoquinones, les anthocyanes, les tanins, les flavonoïdes et des composés phénoliques ( Garaniya et Bapodra, 2014). La richesse de cet extrait en composés chimiques actifs pourrait expliquer l'utilisation traditionnelle d'Abrus precatorius pour soigner de nombreuses maladies telles que la toux et le diabète (Gairola et al., 2010 ; Ezuruike et Prieto, 2014). Des études ont montré que des composés isolés de la plante Abrus precatorius (tanins, alcaloides, sterols, triterpènes et flavonoïdes) possèdent des propriétés antituberculeuse, antiplasmodiale (Limmatvapirat et al., 2004).

L'étude de la toxicité aiguë par voie orale chez les rats, à des doses allant des plus faibles aux plus fortes, n'a présenté aucun mort et aucun signe clinique de toxicité au terme des 14 jours. Ce qui implique que la $\mathrm{DL}_{50}$ est supérieur à $5000 \mathrm{mg} / \mathrm{kg}$ de poids corporel. Selon le système de classification globalement harmonisé de l'OCDE (OCDE, 2001), l'extrait total aqueux des feuilles d'Abrus precatorius peut être classé dans la catégorie 5 et considéré comme une substance non toxique par voie orale. Cette même méthode a été utilisée par Adeneye et Agbaje (2007), qui ont montré que la $\mathrm{DL}_{50}$ de l'extrait aqueux de Cymbopogon citratus (Poaceae) est supérieure à $5000 \mathrm{mg} / \mathrm{kg}$ de pc. Koné et al. 
(2009) par la même méthode, ont montré que la $\mathrm{DL}_{50}$ de l'extrait total aqueux de Sacoglottis gabonensis est supérieur à $5000 \mathrm{mg} / \mathrm{kg}$ pc.

\section{Conclusion}

L'analyse phytochimique qualitative réalisée sur l'extrait total aqueux des feuilles d'Abrus precatorius a montré la présence de plusieurs groupes chimiques, dont la présence dans l'extrait serait responsable de nombreux propriétés thérapeutiques, qui sont attribuées à cette plante. Le test de toxicité aiguë par voie orale chez les rats a montré un effet non toxique aux doses testées. Ces résultats semblent être en faveur de son innocuité par voie orale dans le traitement traditionnel de certaines maladies. Cependant, d'autres travaux tels que de la recherche de l'effet de l'extrait sur certains organes cibles (foie, le rein, le cœur), par le dosage des paramètres biochimiques sériques en toxicité subaiguë ou chronique par voie orale mérite d'être menés afin de vérifier son caractère atoxique.

Une évaluation scientifique des plantes traditionnelles et de leur méthode d'utilisation dans la gestion des maladies, peut permettre leur intégration dans le système de santé officiel en Afrique et dans les autres pays en voix de développement.

\section{REMERCIEMENTS}

Cette étude initiée par le Laboratoire de Pharmacodynamie Biochimique, UFR Biosciences de l'Université Félix Houphouët Boigny Côte d'Ivoire, a bénéficié du concours de plusieurs Laboratoires. Cependant, nous remercions tout particulièrement le Pr. Mostafa KHOUILI Responsable de l'Equipe Chimie Organique Organométallique Appliquée du Laboratoire de Chimie Organique et Analytique, Faculté des Sciences et Techniques, Université Sultan Moulay Slimane de Béni-Mellal (Maroc) pour avoir facilité l'achèvement de cette étude.

\section{REFERENCES}

Adeneye AA, Agbaje EO. 2007. Hypoglycemic and hypolipidemic effects of fresh leaf aqueous extract of Cymbopogon citrates Stapf. in rats. $J$. Ethnopharmacol., 112(3): 440-444.

Gairola S, Gupta V, Bansal P, Singh R, Maithani M. 2010. Herbal antitussives and expectorants. Int. J. Pharm. Sci. Rev. Res., 5(2): 5-6.

Garaniya N, Bapodra A. 2014. Ethno botanical and Phytophrmacological potential of Abrus precatorius L. Asian Pac J Trop Med., 4(1): S27-S34.

Ezuruike UF, Prieto JM. 2014. The use of plants in the traditional management of diabetes in Nigeria: Pharmacological and toxicological considerations. $J$. Ethnopharmacol., 155(2): 859-860.

Houghton PJ, Raman A. 1998. Laboratory Handbook for the Fractionation of Natural Extracts. Ed Chapman and Hall: New York; 208.

Konkon NG, Simaga D, Adjoungova AL, N 'Guessan KE, Zirihi GN, Kone BD. 2006. Etude Phytochimique Demitragyna Inermis (Willd.) O. Ktze (Rubiaceae), plante à feuille antidiabetique. Pharm. Méd. Trad. Afr., 15: 73-74.

Koné M, Bleyere NM, Yapo AP, Vangah MO, Ehilé EE. 2009. Evaluation de la toxicité d'un extrait aqueux de Sacoglottis gabonensis (Baille) Urban (Humiriaceae) chez les rongeurs, une plante utilisée dans le traitement de l'ulcère de Buruli en Côte d'Ivoire. Int. J. Biol. Chem. Sci., 3(6): 1286-1296.

Limmatvapirat C, Sirisopanaporn S, Kittakoop P. 2004. Antitubercular and antiplasmodial constituents of Abrus precatorius. Planta Med., 70(3): 276-278.

N'guéssan K, Zirihi N, Boraud NKM. 2010. Etude ethnopharmacologique des plantes utilisées pour faciliter l'accouchement, en 
pays Abbey et Krobou, au Sud de la Côte-d'Ivoire. Int. J. Biol. Chem. Sci., 4(4): 1009-1010.

OCDE. 2001. Ligne directrice de l'OCDE pour les essais de produits chimiques toxicité orale aiguë - méthode par classe de toxicité aiguë, 14p.

Okoko IE, Yama OE. 2011. Cytoarchitectural variations in the ovary, oviduct and uterus following intra-gastric gavages of Abrus precatorius Linn in albino rats. Int. J. Morphol., 29(4): 1408-1413.

Prenner G. 2013. Flower development in Abrus precatorius (Leguminosae: Papilionoideae: Abreae) and a review of androecial characters in Papilionoideae. South African Journal of Botany, 89: 210211.
Schrire B. 2005. Tribe Abr eae. In Legumes of the World, Lewis G, Schrire B, Mackinder B, Lock M (eds). Royal Botanic Gardens: Kew.

SGH. 2003. système général harmonisé de classification et d'étiquetage des produits chimiques. Toxicité aiguë. Nations Unies New York et Genève, publication des nations unies numéro de vente : F.03.II.E.25, 547p.

Suralkar AA, Kasture SB. 2013. Inhibitory effect of Abrus precatorious extract on bronchial hyper-reactivity induced by ovalbumin in experimental animals. Int. J. Pharm. Pharm. Sci., 5(1): 403-404. 